\title{
A retrospective clinical control study of extended anterolateral approach and Frosch approach in Schatzker type II tibial plateau fractures involving the posterolateral column
}

\section{Yongqing Yan}

University of Chinese Academy of Sciences https://orcid.org/0000-0003-1831-8861

\section{Renjie Xu}

Suzhou Municipal Hospital

Xinhua Yuan ( Fixboner@163.com )

Orthopedics Center, HwaMei Hospital, University of Chinese Academy of Science, Ningbo City, Zhejiang Province, China

\section{Research article}

Keywords: Tibial Plateau Fractures, Posterolateral Column, Approach, Internal Fixation

Posted Date: May 27th, 2020

DOI: https://doi.org/10.21203/rs.3.rs-30667/v1

License: (c) (i) This work is licensed under a Creative Commons Attribution 4.0 International License.

Read Full License 


\section{Abstract}

Objective: To compare the extended anterolateral approach with the Frosch approach in the treatment of Schatzker type II tibial plateau fractures involving the posterolateral column.

Methods: A retrospective case-control study was conducted to compare the general information, intraoperative and postoperative situations, HSS score and Rasmussen radiology score, TPA, and PSA immediately after the operation and at one-year follow-up.

Results: There was no statistical difference between the two groups in general information, which was comparable. The operation time in the Frosch group was slightly higher than those in the control group, while the HSS score and Rasmussen radiology score were better than those in the control group.

Conclusion: In the case with simple posterolateral fragments, the extended anterolateral approach and the Frosch approach can both achieve better outcomes. While in the case of complex posterolateral fragments, the Frosch approach can provide more accurate reduction and better biomechanical stability.

\section{Introduction}

With the popularization of CT and MRI, the incidence of tibial plateau fracture with posterolateral depression has proved not as low as previously considered, which can be even as high as $15 \%-44.2 \%$ of all tibial plateau fracture and $50.35 \%$ of Schatzker Type II fracture(1).

Radiographic studies showed that the area of posterolateral fragment accounts for $15 \%-30 \%$ of the tibial plateau area and the average displacement is more than $3 \mathrm{~mm}(2)$. When the step-off reaches $3 \mathrm{~mm}$, the stress will increase by $75 \%$, which is one of the surgical criteria.

The most direct approach for PLCF is the posterior approach. Although the results of these different approaches with posterior plating fixation are satisfactory so far, in further studies, the risks and shortcomings of the posterior approach are gradually realized(3), especially the iatrogenic injury of normal structures(4). Besides, there is also limited exposure of tibial plateau structures, lack of reference for reduction, the difficulty in internal fixation removal, and so on $(5,6)$.In order to reduce the damage to blood supply and nerves, the posterior reversed L-shaped approach was adopted, but the awkward position and limited exposure for vision could not be avoided during the operation(7, 8). It was reported that the femoral condyle osteotomy approach, the lateral fibular head osteotomy approach, the anterolateral fibular head approach, or the extended anterolateral approach all could improve the exposure(9-11). However, the disadvantage is also that the normal lateral collateral ligament and other structures are iatrogenically injured, which increases the risk of further knee instability and the common peroneal nerve injury.

The anterolateral approach is the primary approach for the treatment of tibial lateral plateau fractures, and it is also the most familiar approach for traumatologists. However, it can only expose $1 / 3$ of the 
lateral plateau. When it comes to PLCF involved, it is more complicated. However, there have been some reports of standard or extended anterolateral approach for the treatment of PLCF, and excellent results have been achieved $(12,13)$. Frosch(14)treated this kind of fracture through a single incision and double space approach and also received excellent results. There is still controversy on the choice of the approach. In this study, two groups of patients were compared.

\section{Materials And Methods}

\section{Inclusion and exclusion criteria}

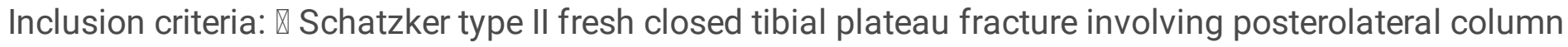
within two weeks after injury; $\forall$ sagittal width of the involved posterolateral tibial plateau articular surface $>1.5 \mathrm{~cm}$; $\otimes$ no injury of nerves, blood vessels,no severe injury of head, chest, abdomen, nor other critical

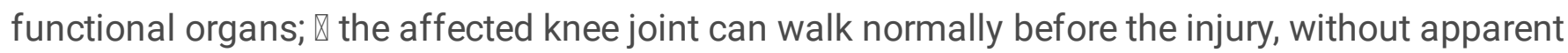
osteoarthritis and trauma history. Exclusion criteria: $\nabla$ open tibial plateau fracture; $\nabla$ accompanying

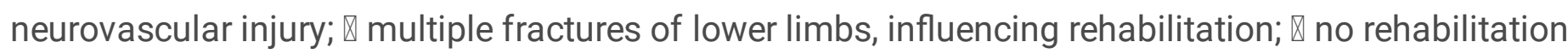
treatment after operation; $\mathbb{\nabla}$ unable to follow up on schedule or follow-up time $<1$ year.

\section{General information}

According to the inclusion and exclusion criteria, the data of 23 patients with tibial plateau type II fracture involving the posterior column from January 2014 to January 2018 were collected, including 16 males and 7 females, aged 26-69 years.

\section{Classification}

All cases were Schatzker type II. According to the three-column classification of Luo (14), the posterolateral column of the tibial plateau was involved.

\section{Treatment}

\section{(1) Preoperative planning}

The affected limb was fixed with plaster or calcaneal traction. Before the operation, routinely X-ray, CT scan, and 3D reconstruction were performed, while MR examination taken if necessary, and operation was performed after 'wrinkle sign'appeared.

\section{(2) Surgical technique}

The extended anterolateral approach group $₫ G$ roup $A \bigotimes(12)$ : First, the limb was slightly flexed. An approximately 15 - cm-long S-shaped incision with Gerdy's tubercle as the center was made to expose iliotibial band and anterolateral calf fascia. The iliotibial band was split in the middle along the direction of the fibers and sharply elevated from Gerdy's tubercle anteriorly and posteriorly. The fascia incision was extended downward, and about $5 \mathrm{~mm}$ fascia flap was left for further repair. The dissection was extended 
posteriorly by taking down the extensor muscles from the lateral surface of the lateral plateau to the point in front of the fibular collateral ligament (FCL). Then, the knee joint was flexed up to $90^{\circ}$ to relax the fibular collateral ligament and the common peroneal nerve. Then, with the FCL retracted posterolaterally, the posterolateral surface of the lateral plateau was exposed by dissecting loose soft tissues between them. Last, we opened the joint capsule and retracted the lateral meniscus superiorly to expose the joint surface. After that, we reduced the fragments and used Kirschner wires for temporary fixation. The lateral locking plate (Synthes GMBH, Zuchwil, Switzerland) was placed as posteriorly as possible, and the transverse arm stretched to the supra-fibular-head space. In an excellent situation, two rafting screws supported the articular surface of the posterolateral column.

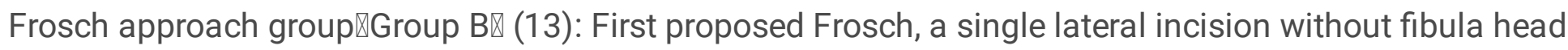
osteotomy was used to treat the anterolateral and posterolateral plateau fractures at the same time. The treatment of anterolateral platform fracture is the same as the previous anterolateral approach. However, at the same time, it enters the exposed posterolateral platform between the lateral head of gastrocnemius muscle and soleus muscle, to achieve an incision treatment of tibial platform fracture involving both the lateral column and the posterolateral column.

Compared to the original Frosch approach, a little modification was made in this practice. The anterolateral fracture was treated in the interval of the anterior tibial muscle. The fibula starting point of the soleus muscle was sharply dissected downward along the fibula, and it was pulled to the posteromedial together with the lateral head of the gastrocnemius muscle to reveal the posterolateral fragments instead of through the interval between the lateral head of the gastrocnemius muscle and the soleus muscle. However, there was no essential difference from the Frosch approach.

\section{Postoperative management}

Postoperative drainage routinely continued for $48 \mathrm{~h}$, and also the antibiotic use. Low molecular weight heparin was used to prevent deep venous thrombosis of the lower limbs, according to the Caprini Score. Early ROM and non-weight bearing movements were emphasized. Passive joint function activity was performed immediately after the operation, and continuous passive motion exercises were initiated on the second day postoperatively. Three days later, the patients were encouraged to participate in active motion rehabilitation without weight-bearing under the guidance and supervision of the surgeons. Partial weightbearing was allowed after the second postoperative week(12), but not the loaded squat. The time to full weight-bearing depended on the radiographic evidence of fracture healing and callus formation.

\section{Data collection and follow-ups}

General information of patients was collected before the operation, as well as medical complications.

The operation time, blood loss, fracture healing time, fracture reduction, the American Hospital For Special Surgery (HSS) score(15), knee range motion, tibial plateau angle(TPA), posterior slope angle(PSA) and postoperative complications were noted. According to the standard clinical and radiological criteria 
to judge the healing time of the fracture, the Rasmussen score(16) system was used to evaluate knee joint function in the 12th month. Patients were followed-up every month until The radiographic evidence showed fracture healing and callus formation. Then patients were followed-up every three months for at least 12 months in total.

\section{Statistical analysis}

The operation time, blood loss, fracture healing time, knee HSS and Rasmussen score, and range motion, and other quantitative data were compared between group $A$ and group $B$ by the independent $t$-test, while categorical data compared by the chi-square test (Fisher's exact test). $\mathrm{P}<0.05$ was considered statistical significant. Graphpad prism 8.02 (GraphPad Software Inc. San Diego) was adopted to record and analyze the study results.

\section{Results}

The general information of the two groups are comparable. (shown in Table 1).

There are operation time, Rasmussen Radiology Score, HSS Score with statistical significance in the two groups. Both groups have one case suffering from fat liquefaction without reoperations (detailed as in Table 1, two typical cases in Fig1 and Fig2).

\section{Discuss}

The injury mechanism of the posterolateral tibial plateau fracture is an axial force with the knee in flexion or semi-flexion state, so that the lateral femoral condyle collides with the posterolateral tibial plateau (17). Three-dimensional reconstruction of CT scans is strongly recommended to be performed before injury assessment and treatment(18). The posterolateral approach of the tibial plateau can expose a relatively small area but also be obstructed by the fibular head. There are common peroneal nerve and blood vessels, which increases operative risk.

\section{Approach}

The anterolateral approach widely used in tibial plateau fracture can not directly get to the collapse in the posterolateral tibial plateau. According to the articles(19), the Surgical Procedures of standard anterolateral approach are not only tricky in operation but also, the average joint surface step-off is up to $5.5 \mathrm{~mm}$. Therefore, some scholars have proposed an expanded anterolateral approach(8), which solves the problems well if a simple posterolateral fragment involved but not with complex or comminuted fragments.

The posterior medial approach reveals the posterior structure of the knee joint through the posterior inverted " $L$ " skin incision and the medial interval between the gastrocnemius and soleus muscles(20). An advantage of this approach is that it gives an excellent vision, but it is difficult to fully expose the 
posterolateral articular surface, especially in patients with strong muscles. Therefore, a combined posteromedial and anterolateral approach under a floating position is often necessary, which increases surgical trauma. Moreover, it is challenging to fix the posterolateral fragment through this approach above the fibular head. However, the direct posterolateral approach (Carlson approach)(21) can reconstruct the posterolateral rim and fix it with a plate directly. However, through this approach, direct vision can not be made to the articular surface, so when the lateral platform involved in a wide area, it will appear inadequate.

The lateral approach of fibular osteotomy is specially designed for the treatment of such fractures. Although some researchers reported the superiority of this approach, some patients have complications such as lateral instability of knee joint, injury of the common peroneal nerve, and nonunion of the fibula(22).

The Frosch approach(23) has the advantages of both the anterolateral approach and posterolateral approach in the treatment of Schatzker Type II tibial plateau fractures involving the posterolateral column. Two surgical operation windows through one skin incision are used to cooperate: the posterolateral window is used to treat the posterolateral wall and place the supporting plate; the lateral window is equivalent to the extended anterolateral approach, with an excellent operative vision, which can reduce the articular surface and plating. Monitored through the anterolateral window, the reduction of the posterolateral fragments can be completed in the posterolateral window. One thing should be paid attention to that the posterior tibial blood vessels and nerves go down between the lateral head of the gastrocnemius and soleus muscle at the medial side of the posterior tibial plateau, and the peroneal artery and vein come out at $5 \mathrm{~cm}$ from the articular which limits the extension of the incision. In our study, a little improvement was made in clinical practice. The anterolateral plateau fracture was operated in the tibialis anterior intermuscular interval. The fibula starting point of the soleus muscle was sharply dissected along the fibula and pulled to the posteromedial side together with the lateral head of the gastrocnemius muscle. However, there was no essential difference from the Frosch approach. In this study, the operation time was slightly prolonged, the blood loss increased, but the number of intraoperative fluoroscopies decreased.

\section{Biomechanics and internal fixation}

Zhang(24) Compared four different fixation methods, found that the posterolateral supporting plate had the best biomechanical stability. The anatomic study showed that(25) the anteroposterior distance of the lateral tibial plateau was about $38 \mathrm{~mm}$. In the traditional anterolateral approach, posterior screws were at least $16 \mathrm{~mm}$ from the rim of the posterolateral plateau, and about $40 \%$ of the posterolateral articular surface remains unsupported by current plate systems (4). In the extended anterolateral approach, the posterolateral fragment can be fixed with two screws by placing the plate posteriorly and crossing over the fibular head, in which the mechanical stability is significantly increased(24), but improvement needs to meet those prerequisites: 1 ) the position of the plate and screw is excellent; 2 ) the posterior fragments 
cannot be too comminuted. Some scholars designed a "magic screw" to increased biomechanics stability(3) but still did not consider the situation of posterior fracture comminution.

Considering the limited space and occlusion of the fibular head, the $2.7 \mathrm{~mm}$ or $2.4 \mathrm{~mm}$ distal radius Tshaped plate might have biomechanical and anatomical advantages: $\triangle$ For its small size, it is more

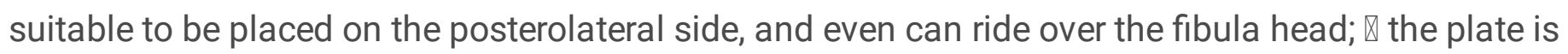
placed close to the joint surface and has excellent angular stability; $\otimes 4-5$ locking screws can be fixed under the joint surface, which has a better rafting effect, and the screw length can be as long as $35 \mathrm{~mm}$; $\mathbb{}$ The suture holes can be used to repair the posterior meniscus and joint capsule. There is no direct evidence of biomechanics stability by the present. However, according to the existing studies(26), Ao 2.4 $\mathrm{mm}$ distal radius volar locking plate can carry a maximum load of more than $1500 \mathrm{~N}$. According to the past rehabilitation experience, patients with tibial plateau fractures should not have full weight knee flexion(3) before fracture healing. Even during weight flexion and extension, the maximum load of the knee joint is about four to five times the weight of patients, and the ratio of the medial compartment to lateral is about 1.7 times(27). Based on the rough estimation of a $60 \mathrm{~kg}$-weighted patient, the requirement of the posterior plate is about $1100 \mathrm{~N}$ at maximum load, which is no more than the maximum load of 2.4 system. In our study, there was no rapture of plate and loss of reduction during the follow-up. It also showed that the scores of HSS and Rasmussen radiology are slightly better than the control group. The poor postoperative scores of the extended anterolateral approach group in the case of the comminuted posterolateral fragment might also be the reason why the scores were lower in the extended anterolateral approach group. Besides, some scholars have further proposed new strategies to fix posterolateral depression in tibial plateau fractures using "Barrel hoop plate" technique(23).

\section{Summary}

In general, as for a simple posterolateral fragment, both the extended anterolateral approach and the Frosch approach can obtain good fixation. The extended anterolateral approach may have higher requirements for techniques of reduction and plating, but it is of convenience in anatomy and fixation removal. While comminuted posterolateral fragments concerned, the Frosch approach can provide more accurate reduction and firm fixation. This study is a retrospective study, and there are some disadvantages, such as no random method, the bias in selection, small sample.

\section{Declarations}

\section{Acknowledgments}

Thanks to our department for help with this study.

\section{Funding}

Supported by Natural Science Foundation of Ningbo Municipality $₫ 2016$ A610142】, Medical Scientific Research Foundation of Zhejiang Province, China(2019KY602) and Young medical key talent of Jiangsu 
Province $₫$ ONRC2016242》

\section{Availability of data and materials}

The datasets used or analyzed during the current study are available from the corresponding author on reasonable request.

\section{Authors' contributions}

YQY collected the patients' data and was a major contributor in writing the manuscript. XHY performed the surgery, gave the idea, and made patients' follow-up checks. RJX took part in designing the study, translating it into English and data analysis. All authors read and approved the final manuscript and the author's order.

\section{Ethics approval and consent to participate}

Ethical approval was obtained by the ethics committee of HwaMei Hospital, University of Chinese Academy of Science. All consents to participate were obtained from the participants.

\section{Consent for publication}

We have consent for publication for every patient in our series.

\section{Competing interests}

The authors declare that they have no competing interests.

\section{References}

1. Zhai Q, Luo C, Zhu Y, et al. Morphological characteristics of split-depression fractures of the lateral tibial plateau (Schatzker type II): a computer-tomography-based study. Int Orthop. 2013;37:911-917.

2. Shen QJ, Zhang JL, Xing GS, et al. Surgical Treatment of Lateral Tibial Plateau Fractures Involving the Posterolateral Column. Orthop Surg. 2019;11:1029-1038.

3. Sun H, Zhu Y, He QF, et al. Reinforcement strategy for lateral rafting plate fixation in posterolateral column fractures of the tibial plateau: The magic screw technique. Injury. 2017;48:2814-2826.

4. Sun $H, H e Q F$, Zhang BB, et al. A biomechanical evaluation of different fixation strategies for posterolateral fragments in tibial plateau fractures and introduction of the 'magic screw'. Knee. 2018;25:417-426.

5. Chang SM, Zheng HP, Li HF, et al. Treatment of isolated posterior coronal fracture of the lateral tibial plateau through posterolateral approach for direct exposure and buttress plate fixation. Arch Orthop Trauma Surg. 2009;129:955-962.

6. Huang YG, Chang SM. The posterolateral approach for plating tibial plateau fractures: problems in secondary hardware removal. Arch Orthop Trauma Surg. 2012;132:733-734. 
7. Qiu WJ, Zhan Y, Sun H, et al. A posterior reversed L-shaped approach for the tibial plateau fractures-A prospective study of complications (95 cases). Injury. 2015;46:1613-1618.

8. Sun H, Zhai QL, Xu YF, et al. Combined approaches for fixation of Schatzker type II tibial plateau fractures involving the posterolateral column: a prospective observational cohort study. Arch Orthop Trauma Surg. 2015;135:209-221.

9. Hu SJ, Chang SM, Zhang YQ, et al. The anterolateral supra-fibular-head approach for plating posterolateral tibial plateau fractures: A novel surgical technique. Injury. 2016;47:502-507.

10. Yoon YC, Sim JA, Kim DH, et al. Combined lateral femoral epicondylar osteotomy and a submeniscal approach for the treatment of a tibial plateau fracture involving the posterolateral quadrant. Injury. 2015;46:422-426.

11. Kfuri M, Schatzker J, Castiglia MT, et al. Extended Anterolateral Approach for Complex Lateral Tibial Plateau Fractures. J Knee Surg. 2017;30:204-211.

12. Jiang L, Zheng Q, Pan Z. Comparison of extended anterolateral approach in treatment of simple/complex tibial plateau fracture with posterolateral tibial plateau fracture. J Orthop Surg Res. 2018;13:303.

13. Gavaskar AS, Gopalan H, Tummala NC, et al. The extended posterolateral approach for split depression lateral tibial plateau fractures extending into the posterior column: 2 years follow up results of a prospective study. Injury. 2016;47:1497-1500.

14. Frosch $\mathrm{KH}$, Balcarek $\mathrm{P}$, Walde $\mathrm{T}$, et al. A new posterolateral approach without fibula osteotomy for the treatment of tibial plateau fractures. J Orthop Trauma. 2010;24:515-520.

15. Insall JN, Dorr LD, Scott RD, et al. Rationale of the Knee Society clinical rating system. Clin Orthop Relat Res. 1989:13-14.

16. Rasmussen PS. Tibial condylar fractures. Impairment of knee joint stability as an indication for surgical treatment. J Bone Joint Surg Am. 1973;55:1331-1350.

17. Xie X, Zhan Y, Wang Y, et al. Comparative Analysis of Mechanism-Associated 3-Dimensional Tibial Plateau Fracture Patterns. J Bone Joint Surg Am. 2020;102:410-418.

18. Van den Berg J, Struelens B, Nijs S, et al. Value of three-dimensional computed tomography reconstruction in the treatment of posterior tibial plateau fractures. Knee. 2020;27:3-8.

19. Solomon LB, Stevenson AW, Lee YC, et al. Posterolateral and anterolateral approaches to unicondylar posterolateral tibial plateau fractures: a comparative study. Injury. 2013;44:1561-1568.

20. Krause M, Kruger S, Muller G, et al. How can the articular surface of the tibial plateau be best exposed? A comparison of specific surgical approaches. Arch Orthop Trauma Surg. 2019;139:13691377.

21. Carlson DA. Posterior bicondylar tibial plateau fractures. J Orthop Trauma. 2005;19:73-78.

22. Pires RES, Giordano V, Wajnsztejn A, et al. Complications and outcomes of the transfibular approach for posterolateral fractures of the tibial plateau. Injury. 2016;47:2320-2325. 
23. Yi Z, Hui S, Binbin Z, et al. A new strategy to fix posterolateral depression in tibial plateau fractures: Introduction of a new modified Frosch approach and a "Barrel hoop plate" technique. Injury. 2020.

24. Zhang W, Luo CF, Putnis S, et al. Biomechanical analysis of four different fixations for the posterolateral shearing tibial plateau fracture. Knee. 2012;19:94-98.

25. Sassoon AA, Torchia ME, Cross WW, et al. Fibular shaft allograft support of posterior joint depression in tibial plateau fractures. J Orthop Trauma. 2014;28:e169-175.

26. Levin SM, Nelson CO, Botts JD, et al. Biomechanical evaluation of volar locking plates for distal radius fractures. Hand $(N$ Y). 2008;3:55-60.

27. Escamilla RF. Knee biomechanics of the dynamic squat exercise. Medicine and science in sports and exercise. 2001;33:127-141.

\section{Table}

Table 1 Comparison of two groups 
Extended Anterolateral Group

\begin{tabular}{|c|c|c|c|c|}
\hline Age & $51.4 \pm 13.0$ & $52.6 \pm 13.6$ & -0.219 & 0.828 \\
\hline $\operatorname{Gender}\left(x^{2}\right)$ & $\mathrm{F} 4 / \mathrm{M} 8$ & F3/M8 & 0.100 & 0.752 \\
\hline Operation Time(Min) & $149.7 \pm 19.9$ & $164.8 \pm 12.2$ & 2.169 & 0.042 \\
\hline Blood Loss(mL) & $240.4 \pm 39.2$ & $272.7 \pm 46.6$ & 1.804 & 0.086 \\
\hline Follow-Up Time(Months) & $22.9 \pm 6.3$ & $23.4 \pm 6.2$ & -0.206 & 0.839 \\
\hline $\begin{array}{l}\text { Intraoperative } \\
\text { Fluoroscopy(Times) }\end{array}$ & $10.8 \pm 3.2$ & $10.9 \pm 2.6$ & 0.062 & 0.951 \\
\hline Healing Time(Months) & $12.6 \pm 1.5$ & $12.5 \pm 1.2$ & 0.066 & 0.948 \\
\hline Full Weight-Bearing (Months) & $17.7 \pm 2.8$ & $16.4 \pm 3.1$ & 0.988 & 0.334 \\
\hline Knee Flexion $\left({ }^{\circ}\right)$ & $125.4 \pm 5.4$ & $124.1 \pm 5.8$ & 0.560 & 0.578 \\
\hline Knee Extension( $\left(^{\circ}\right)$ & $2.3 \pm 2.3$ & $2.0 \pm 1.9$ & 0.380 & 0.708 \\
\hline HSS Score & $91.2 \pm 3.1$ & $93.9 \pm 2.6$ & -2.206 & 0.039 \\
\hline TPA-Postoperative $\left({ }^{\circ}\right)$ & $85.7 \pm 4.4$ & $86.3 \pm 3.4$ & 0.329 & 0.746 \\
\hline TPA-12 Months $\left({ }^{\circ}\right)$ & $86.0 \pm 3.5$ & $85.0 \pm 4.1$ & -0.655 & 0.520 \\
\hline PSA-Postoperative $\left({ }^{\circ}\right)$ & $8.4 \pm 1.7$ & $8.7 \pm 1.2$ & 0.447 & 0.659 \\
\hline PSA-12 Months $\left({ }^{\circ}\right)$ & $9.9 \pm 1.4$ & $9.2 \pm 1.3$ & -1.236 & 0.230 \\
\hline Rasmussen Radiology Score & $13.8 \pm 2.2$ & $15.6 \pm 1.7$ & 2.183 & 0.041 \\
\hline Complication & One fat liquefaction & $\begin{array}{l}\text { One fat } \\
\text { liquefaction }\end{array}$ & & \\
\hline
\end{tabular}

Figures 

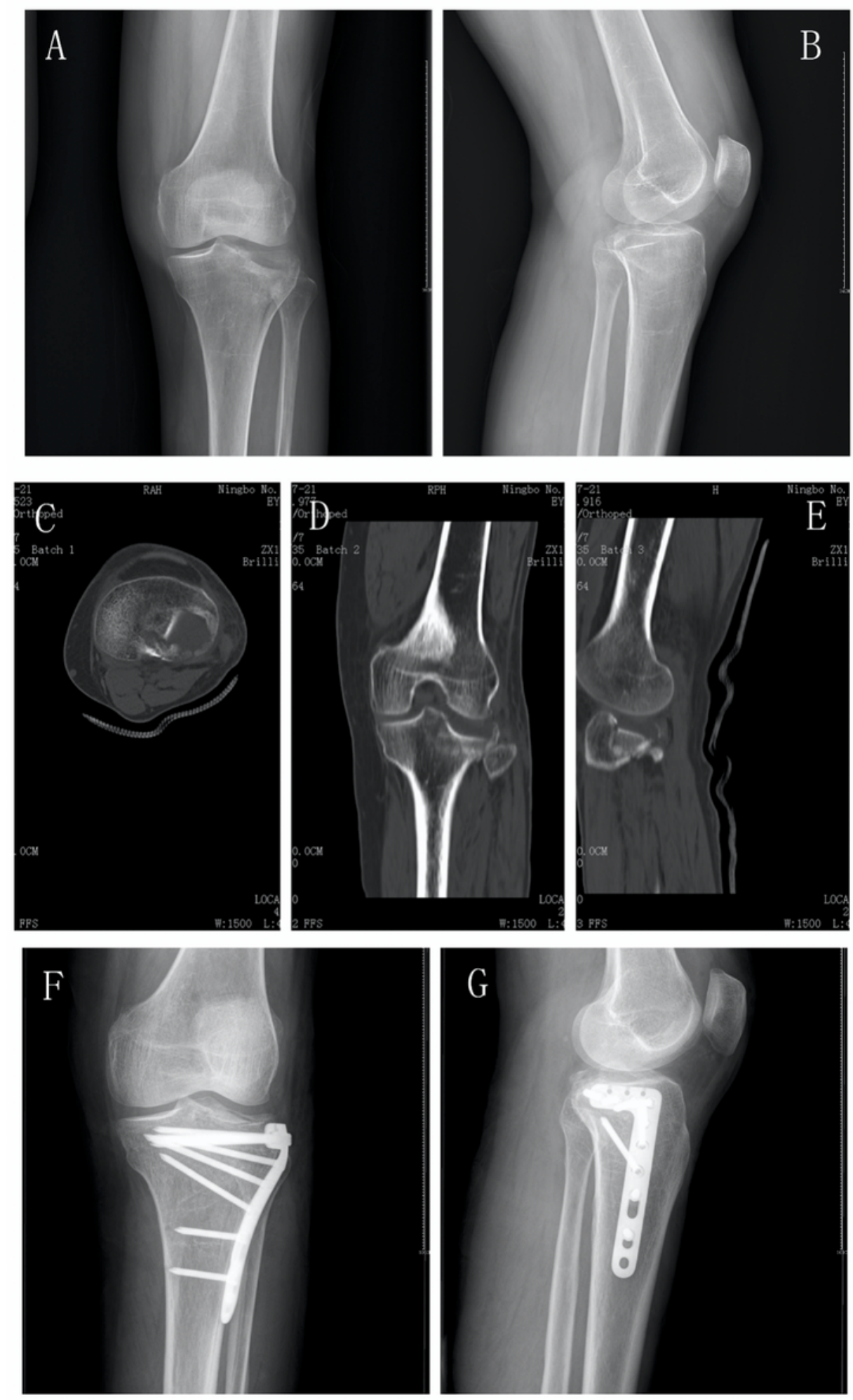

\section{Figure 1}

A 63-year old female, injured in a traffic accident. Preoperative X-ray (A, B) and CT(C, D, E) showed lateral plateau fracture and posterolateral fracture with a relatively simple fragment. Postoperative $X$-ray $(F$, $\mathrm{G})$ showed that the fracture was anatomically reduced and fixed by a lateral locking plate with rafting screws. 

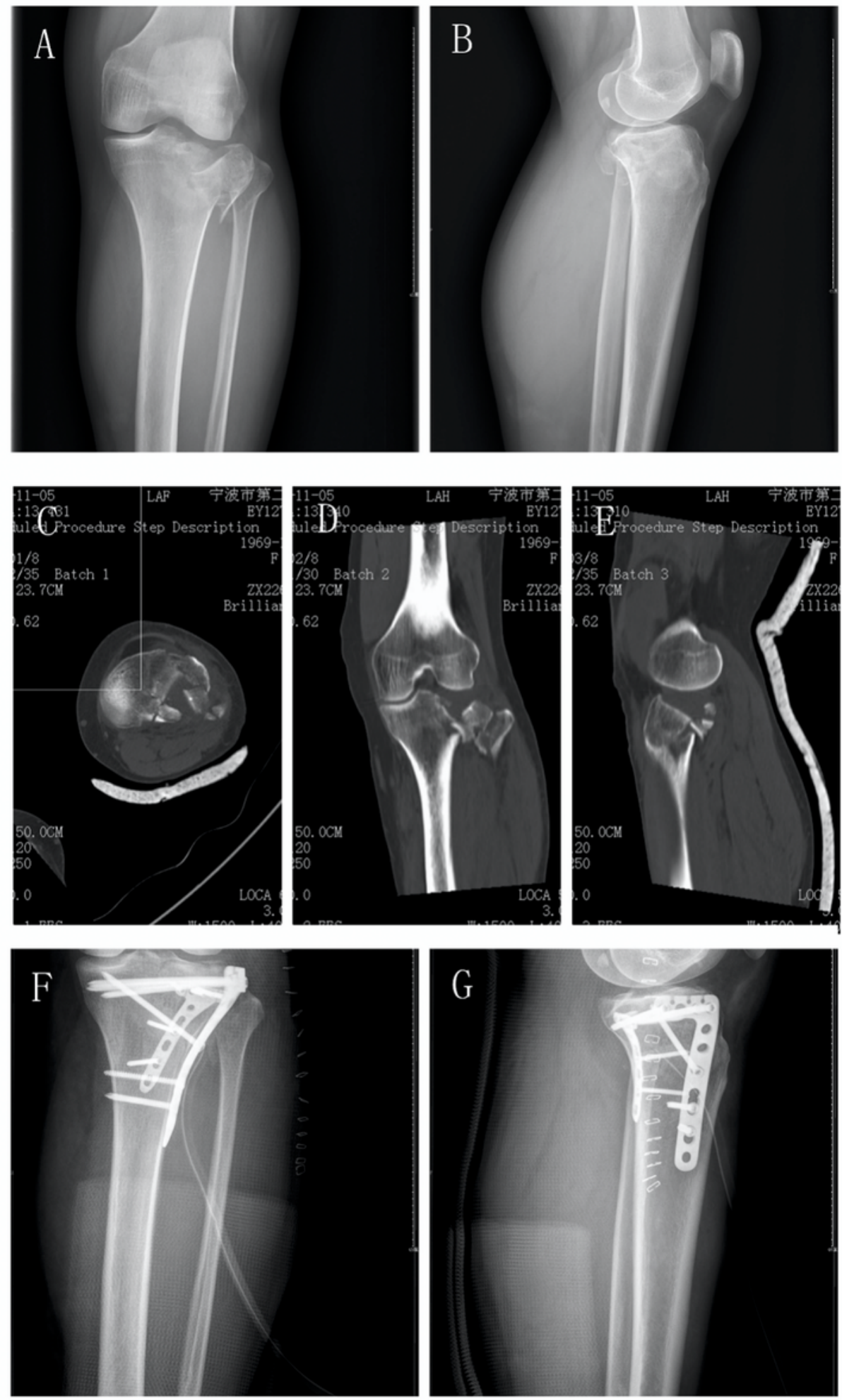

\section{Figure 2}

A 49-year old female injured in a traffic accident. Preoperative X-ray (A, B) and CT(C, D, E) showed lateral plateau fracture and posterolateral fracture with comminuted fragments. Postoperative X-ray $(\mathrm{F}$, $\mathrm{G}$ )showed that the fracture was anatomically reduced and fixed by a lateral locking plate and a $2.7 \mathrm{~mm}$ distal radius locking plate. The patient suffered from fat liquefaction postoperatively but resulted in delayed healing without a reoperation 